\title{
Adaptive Control Algorithm to Provide Bus Priority with a Pre-signal
}

\author{
Haitao He ${ }^{a, *}$, S. Ilgin Guler, Ph.D. ${ }^{b}$, Monica Menendez, Ph.D. ${ }^{a}$ \\ ${ }^{a}$ Traffic Engineering Group, Institute for Transport Planning and Systems, ETH Zurich, Switzerland \\ ${ }^{b}$ Department of Civil and Environmental Engineering, The Pennsylvania State University, USA
}

${ }^{*}$ Corresponding author

Phone: +41 /(0)44-633-3195

Email address: haitao.he@ivt.baug.ethz.ch (Haitao He)

(C) 2016. This manuscript version is made available under the Elsevier user license http://www.elsevier.com/open-access/userlicense/1.0/ 


\begin{abstract}
In urban areas, where road space is limited, it is important to provide efficient public and private transportation systems to maximize person throughput, for example from a signalized intersection. To this end, this research looks at providing bus priority using a dedicated bus lane which is terminated upstream of the intersection, and placing an additional signal at this location, called a pre-signal. Although pre-signals are already implemented in some countries (e.g. UK, Denmark, and Switzerland), an adaptive control algorithm which responds to varying traffic demands has not yet been proposed and analyzed in the literature. This research aims to fill that gap by developing an adaptive control algorithm for pre-signals tailored to real-time private and public transportation demands. The necessary infrastructure to operate an adaptive pre-signal is established, and guidelines for implementation are provided. The relevant parameters regarding the boundary conditions for the adaptive algorithm are first determined, and then quantified for a typical case using a micro-simulation model. It is demonstrated with case studies that, under all considered scenarios, implementing a pre-signal with the proposed adaptive control algorithm will result in the least average person delay at the intersection. The algorithm is expected to function well with a wide range of car demands, bus frequencies, and bus passenger occupancies. Moreover, the algorithm is robust to errors in these input values, so exact information is not required.
\end{abstract}


Keywords: Pre-signal; Bus Lane; Transit Priority; Public Transport; Adaptive Control 


\section{Introduction}

Providing priority to public transport (e.g. buses) in cities could reduce travel times of buses. Often, this kind of priority is provided with a dedicated bus lane. However, this might not always be feasible due to space restrictions or political issues. It might not be the most efficient solution either, especially if the bus flow is low, the private vehicle (e.g. car) demand is high, or bottlenecks such as traffic signals exist. Under such situations, alternative strategies could be proposed to provide bus priority while minimizing negative impacts on cars.

One such alternative strategy to provide bus priority at a signalized intersection where a dedicated bus lane exists, is to terminate the dedicated bus lane upstream of the main signal and place an additional signal at this location. This additional signal only controls cars and is hereafter referred to as a pre-signal (Wu and Hounsell, 1998: Guler and Menendez, 2013, 2014a b). Its configuration is illustrated in Figure 1a. The primary purpose of the pre-signal is to allow buses to jump the car queues upstream of the intersection while cars can still use all the lanes at the main signal to fully utilize the capacity of the intersection. The distance between the pre-signal and the main signal is long enough to allow a queue of the required number of cars to saturate the green at the main signal for an over saturated scenario. In this way, bus delays are reduced, while the capacity loss at the intersection is minimized.

Some pre-signals are installed around the world (e.g. UK, Denmark, and Switzerland) with slightly different operating strategies. This paper assumes a previously proposed operating strategy (Guler and Menendez, 2014a). In this strategy, the pre-signal turns red for cars in advance of a red main signal by the free-flow travel time, and also when a bus arrives to the pre-signal irrespective of the status of the main signa 1 . The goal is to ensure that the space between the pre-signal and the main signal is kept free of cars for as long as possible, allowing any arriving bus to move in front of the car queue and discharge immediately when the main signal turns green. The pre-signal turns green for cars more than the free-flow travel time in advance of the main signal when no buses are present, such that cars do not experience additional delays, or after the bus has left the pre-signal when buses are present. Note that the pre-signal does not affect the operation of the main signal.

The commonly used strategy of extending the dedicated bus lane up to the main signal is another alternative (hereafter termed the continuous bus lane strategy, see Figure 1b). This could further reduce the bus delay, and hence the average person delay if the bus occupancy or the bus frequency is high. Another feasible option is to still terminate the bus lane upstream of the main signal, however, do not apply any control to cars at this point and allow buses and cars to freely merge (hereafter termed the interrupted bus lane strategy, see Figure 1. ). This could further increase the traffic throughput and reduce the upstream queue length when traffic demand is high. Notice that the basic configuration of the road section upstream of the pre-signal location is the same across the three strategies.

[Figure 1 about here.]

The purpose of this research is to propose an adaptive control algorithm for pre-signals tailored to real time private and public transportation demands. To do so, this research establishes the analytical form of the boundary conditions (based on the car demand, bus occupancy, and bus frequency) for the implementation of the adaptive control algorithm. These boundary conditions determine when each of the three strategies considered would benefit the system the most. Using these boundary conditions, the control at the presignal is determined in real-time. There are two levels of control. The first level is the signal timing control

\footnotetext{
${ }^{1}$ The pre-signal turning red for any bus arrival may not always save bus delays, while it will always impart additional car delays. However, this basic strategy is implemented since it reduces the required inputs to the algorithm, more specifically the knowledge of the existence of a queue at the main signal. Hence, this is a conservative assumption resulting in the car delays representing the worst-case scenario for the pre-signal strategy.
} 
algorithm for the operation of the pre-signal. However, continuously operating the pre-signal might not be optimal based on the boundary conditions. Hence, the two alternative strategies described above (the continuous bus lane strategy or the interrupted bus lane strategy) could be adopted by making the pre-signal continuously green, or using dynamic message signs. Switching between these strategies is possible because it only involves changes in lane allocation for a small portion of the road immediately upstream of the main signal. Hence, the second level control proposes an algorithm to switch between different strategies, based on real time traffic inputs. In this way, traffic operations at the intersection can be controlled dynamically in response to car and bus demand variations throughout the day.

The remainder of the paper is organized as follows. Section 2 reviews previous literature on pre-signals. Section 3 establishes the objective function and constraint for optimizing the operations at an intersection under relevant conditions. Section 4 proposes the first level control algorithm for the operation of the presignal. Section 5 uses a calibrated and validated micro-simulation model to establish the boundary conditions for the operation of the pre-signal, and tests their sensitivity to several parameters. Section 6 proposes the second level (adaptive) control algorithm, illustrates its use with three case studies, and tests its robustness to errors in the input data. Lastly, Section 7 offers some concluding remarks.

\section{Literature review}

The idea of setting up a signal in front of the main signal (i.e. a pre-signal) is not new and has been studied before. Pre-signals have been proposed for several purposes, such as to recover the lost time at the intersection due to bounded acceleration (Von Stein, 1961), to separate left-turning vehicles and throughmoving vehicles to maximize the discharging capacity of the intersection for both directions (Xuan et al. 2011), or to provide priority to buses using the opposite lane (Guler et al., 2015, 2016). This paper, however, studies pre-signals used to provide priority to buses without using the opposite lane.

The use of pre-signals to provide bus priority was first proposed by $\mathrm{Wu}$ and Hounsell (1998). This work also provided guidelines to determine the pre-signal timing for minimizing the system delay. However, the proposed operating strategy was for intersections without bus detection infrastructure. This is implemented in a few cases in U.K. cities, for example in London (Transport for London, 2009). Sometimes, bus gates with signals can also refer to a similar strategy (Department for Transport (U.K.), 2003). A comparable idea for pre-signals is also mentioned in the German Manual for Transit Priority (Forschungsgesellschaft für Straßen-und Verkehrswesen, 1991). Although pre-signals are already implemented in some countries, to the authors' best knowledge, most existing pre-signals are implemented with empirically determined control algorithms. Therefore, there is a need to propose a general adaptive algorithm and to study its effects.

The operating strategy described in the introduction and used in the rest of this paper was first studied empirically (Guler and Menendez, 2013) and then examined analytically (Guler and Menendez, 2014a b) for an isolated intersection. Queuing theory was used in the formulation assuming uniform demands. It was concluded that, for under-saturated demands (less than $85 \%$ of the signal capacity), the pre-signal strategy could provide the lowest average person delay for bus occupancies of 10-70 passengers, compared to using mixed lanes or a dedicated bus lane for the whole road section upstream of the main signal. For oversaturated demands with bus occupancies greater than 20, the pre-signal strategy always resulted in lower average person delay compared to a dedicated bus lane. Moreover, pre-signals resulted in lower average person delay compared to mixed lanes if the peak demand was greater than $105 \%$ of the signal capacity. However, the emphasis of previous studies was more to demonstrate the potential benefits of a pre-signal for some general scenarios rather than to establish the exact boundary values when such benefits would be significant. For example, due to the tediousness of the theoretical calculations, a fixed bus frequency of 2-3 minutes/bus was assumed in the analysis (Guler and Menendez, 2014a|b).

However, it is not yet clear if installing a pre-signal is worthwhile for a larger range of demand patterns (including car demand, bus frequency, and bus occupancy) at an intersection. These boundary conditions 
are difficult to solve analytically, especially when accounting for realistic driving behaviours and demand patterns. The queuing theory formulation is not able to account for delays due to lane changes between the pre-signal and the main signal (e.g., lane changing maneuvers which may lead to certain lanes being underutilized by cars). Also, in practice, cars and buses have bounded acceleration and the drivers have a reaction time to changes in the signal. Lastly, a realistic demand at an urban intersection varies greatly with time. These factors are difficult to examine analytically and could be more accurately studied by simulations.

\section{Description of the problem}

For the reader's convenience, a list with the notation used in the algorithm is summarized in Table 1 .

[Table 1 about here.]

In this paper, a bi-level control algorithm is developed for a pre-signal upstream of a signalized intersection. The first level algorithm controls the operation of the pre-signal itself. The second level control combines multiple strategies to respond to varying traffic demands. The geometric configuration of the signalized intersection is as illustrated in Figure 1 $1 \mathrm{a}$. Assume the road has $\lambda$ lanes upstream of the intersection. Each lane has a capacity $\mu$, jam density $k_{j a m}$, and free flow speed $v_{c}$. The fundamental diagram is assumed to be triangular. The main signal at the intersection $(S)$ has an effective green time of $G$ and a cycle length of $\tau$. The effective discharge capacity when all lanes are used at the intersection is,

$$
\mu_{e f f}=\frac{\lambda \mu G}{\tau}
$$

A bus lane runs throughout the whole road section up to the pre-signal $(P)$, which is a distance $\overline{P S}$ upstream of the main signal. The pre-signal operates with the operating strategy described in the introduction. It has a red signal duration of $R_{p}$ when a bus is not detected. $R_{p}$ depends on the car demand. A loop detector is placed a distance $\overline{O P}$ upstream of the pre-signal at $O$. When a bus is detected at the loop detector, the pre-signal will turn red for a duration of $R_{b}$. More details about the road configuration and pre-signal timings will be given in the next section.

Vehicles (either cars or buses) detected at the loop detector are indexed with $k$. Vehicle $k$ carries $X_{k}$ people, passes $O$ at $t_{O k}$, and leaves $S$ at $t_{S k}$. Among the detected vehicles, cars are indexed with $i \in I, I \subseteq K$, and buses are indexed with $j \in J, J \subseteq K$. Cars have free-flow speed $v_{c}$, so the free-flow travel time from $O$ to $S$ is $\overline{O S} / v_{c}$. Buses have free-flow speed $v_{b}$, so the free-flow travel time from $O$ to $S$ is $\overline{O S} / v_{b}$.

The loop detector feeds the detected demand into the pre-signal algorithm every signal cycle indexed $n$. This information is used to determine the red signal duration $R_{p}$. The loop detector also aggregates detection results over a period $\Omega$ corresponding to $N$ successive signal cycles indexed $m$. This information is used to determine the strategy applied at the intersection for the next strategy period, $m+1$. Denote the $n$th signal cycle within the $m$ th strategy period as $T_{m, n}=\left[t_{m, n-1}, t_{m, n}\right]\left(1 \leq n \leq N, t_{m, n}-t_{m, n-1}=\tau\right.$, $\left.t_{m, N}-t_{m, 0}=\Omega=N \tau\right)$. In the $m$ th strategy period $\left[t_{m, 0}, t_{m, N}\right], I_{m}=\left|\left\{i \mid t_{O i} \in\left[t_{m, 0}, t_{m, N}\right]\right\}\right|$ corresponds to the number of cars detected and $J_{m}=\left|\left\{j \mid t_{O j} \in\left[t_{m, 0}, t_{m, N}\right]\right\}\right|$ corresponds to the number of buses detected. $\alpha_{m}$ is the the car volume to capacity ratio (hereafter refer to as the $\mathrm{V} / \mathrm{C}$ ratio) over the $m$ th strategy period,

$$
\alpha_{m}=\frac{I_{m}}{\Omega \mu_{e f f}}
$$

The car volume to capacity ratio over the $n$th signal cycle, $\alpha_{m, n}$, can be calculated similarly. 
To set the objective function and constraint for the traffic operations at the intersection, the furthest distance of perturbation and the average person delay are selected as the determining criteria.

- Criteria \#1. The furthest distance of perturbation $L_{q}$ should not exceed a pre-defined distance $L_{q}^{\max }$ upstream of $S$. When the perturbation reaches too far, the upstream intersection and the performance of the network are likely affected due to spillback effects, as will be discussed below.

- Criteria \#2. The average person delay, $d$, for all the passengers on buses and cars leaving the main signal should be minimized. This metric provides a good balance between car delay and bus delay so that public transport is promoted while car operation is not severely affected.

The distance of the perturbation $L_{q}$ is determined by the car furthest upstream that is not in a free-flow state. Note that this does not mean a static queue of length $L_{q}$ exists. In practice, the distance $L_{q}^{\max }$ should be chosen by an estimate of when such a disruption would be unacceptable. The exact cut-off distance depends on the characteristics of the arterial and is beyond the scope of this paper, as the discussion is limited to an isolated intersection.

The two criteria above could be summarized into the following mathematical program:

$$
\min _{\text {strategy at } P \text { and }} \overline{P S} d=\frac{\sum_{i}\left[\left(t_{S i}-t_{O i}\right)-\frac{\overline{O S}}{v_{c}}\right] X_{i}+\sum_{j}\left[\left(t_{S j}-t_{O j}\right)-\frac{\overline{O S}}{v_{b}}\right] X_{j}}{\sum_{i} X_{i}+\sum_{j} X_{j}}
$$

subject to $L_{q} \leq L_{q}^{\max }$.

\section{First level control algorithm: operation of the pre-signal}

First, we specify the first level control algorithm for the pre-signal. The distance $\overline{P S}$ is given by Guler and Menendez, 2015,

$$
\overline{P S}=\frac{G \mu}{k_{j a m}}
$$

such that there is enough storage space to saturate the main signal when it is green. The pre-signal should not cause extra delay to cars during cycles where buses are not present, so the last discharged car from the pre-signal must pass the main signal within the same cycle. Therefore, the pre-signal has the same cycle length as the main signal with a red signal advance time of $\tau_{\Delta}$ (Guler and Menendez, 2015),

$$
\tau_{\Delta}=\frac{\overline{P S}}{v_{c}} .
$$

Recall that the length of the red time at the pre-signal $\left(R_{p}\right)$ is a function of the car demand $\alpha_{m, n}$ (Guler and Menendez, 2015),

$$
R_{p}\left(\alpha_{m, n}\right)= \begin{cases}\frac{\tau-\alpha_{m, n} \lambda G /(\lambda-1)}{\tau-\alpha_{m, n} G}(\tau-G) & \text { for } \alpha_{m, n}<1 \\ \tau-\lambda G /(\lambda-1) & \text { for } \alpha_{m, n} \geq 1\end{cases}
$$


This is the longest possible red for the pre-signal such that the flow through the main signal would not be affected. The pre-signal operation depends on the demand for $\alpha_{m, n}<1$. Hence, the location of the loop detector is especially important to detect the demand in under-saturated conditions. This location can be determined by considering the maximum reach of the perturbation due to the operation of the pre-signal. The perturbation distance measured from the main signal for an under-saturated cycle $\left(\alpha_{m, n}<1\right)$ is,

$$
L_{q}\left(\alpha_{m, n}\right)=\overline{P S}+R_{p}\left(\alpha_{m, n}\right) k_{j a m} /\left(\frac{1}{\alpha_{m, n} \mu_{e f f}}-\frac{1}{\mu}\right) \text { for } \alpha_{m, n}<1
$$

It could then be derived from Eq.(6) and Eq.(7) that

$$
L_{q}\left(\alpha_{m, n}\right) \leq \overline{P S}+\frac{\left(\tau-\frac{\lambda G}{\lambda-1}\right) k_{j a m}}{\frac{1}{\mu_{e f f}}-\frac{1}{\mu}}, \quad \forall\left(\alpha_{m, n}<1\right)
$$

Also, to set the correct pre-signal timing for a given signal cycle $T_{m, n}$, the loop detector should be placed far enough such that the demand is detected in the prior signal cycle $T_{m, n-1}$. This can be ensured by,

$$
\overline{O P} \geq v_{c} \tau \wedge \overline{O P} \geq v_{b} \tau
$$

To summarize, the requirement for the loop detector placement is,

$$
\overline{O S} \geq \frac{G \mu}{k_{j a m}}+\max \left\{\frac{\left(\tau-\frac{\lambda G}{\lambda-1}\right) k_{j a m}}{\frac{1}{\mu_{e f f}}-\frac{1}{\mu}}, v_{c} \tau, v_{b} \tau\right\}
$$

Notice that this location may be too far from the main signal to be feasible for a loop detector implementation. In this case, the loop detector should be placed as far from the main signal as possible such that the input demands can be fairly accurately determined. However, this will lead to a lag in demand detection as demand for a cycle cannot be predicted in real time while the cycle is being optimized. The effects of this lag on the operation are tested in Section 6.4.

The first level of the control algorithm for the operation of the pre-signal when it is on, is summarized in the flow chart in Figure 2 .

[Figure 2 about here.]

\section{Boundary conditions}

To choose among the three strategies, denote the resulting average person delays when the pre-signal strategy, the interrupted bus lane strategy, and the continuous bus lane strategy are used as $d(1), d(2)$, and $d(3)$, respectively. Then, the solution to the mathematical program shown in Eq. 3 will have a form,

$$
d_{\min }=d(z) ; z \in\{1,2,3\}
$$

with a set of boundary conditions on: 
- V/C ratio: $\alpha_{m}=\frac{I_{m}}{\Omega \mu_{e f f}}$

- bus frequency: $f_{m}=\frac{J_{m}}{\Omega}$

- bus to car passenger occupancy ratio: $\beta_{m}=\frac{\sum_{j} X_{j} / J_{m}}{\sum_{i} X_{i} / I_{m}}$

The boundary conditions establish when each strategy should be adopted. While some of these boundary conditions could be analytically determined using simple kinematic wave theory, these models would not be able to account for factors such as bounded acceleration, reaction time, lane changes, stochastic speeds and stochastic demands. A lack of these factors in the models could lead to differences between the actual and theoretically determined boundary conditions. Unfortunately, these factors cannot be easily accounted for in analytical models because the objective function and constraints would become non-linear and multivariate. Therefore, a micro-simulation model using VISSIM was built to solve this problem. A simulation model is advantageous because it can account for the driver and vehicle factors, examine different performance metrics (other than average person delay), and emulate realistic demand patterns which vary throughout the day. For the simulation to represent a realistic scenario as closely as possible, it was calibrated and validated based on empirical data at a pre-signal in Zurich, Switzerland. The calibration dataset included over 50 cycles of traffic data, and the validation dataset included 15 cycles of traffic data. The details of the calibration and validation process can be found in (He et al., 2014). Notice that some errors in mimicking real traffic would still remain even after the extensive calibration and validation processes. However, these inaccuracies are expected to be small and systematic, and hence, should only minimally influence the results shown in this paper.

The layout of the intersection in the simulation model is exactly as illustrated in Figure1 Table 2 summarizes the variables used in this simulation model (assumed or calculated).

[Table 2 about here.]

The purpose of this simulation model is to establish the form of the boundary conditions (i.e. the important variables, and whether the bounds are upper or lower bounds). Bounds for typical conditions will also be quantified. To do so, the effects of the three strategies under different levels of $\mathrm{V} / \mathrm{C}$ ratios $\left(\alpha_{m}\right)$ are considered. The simulations are run for $12 \mathrm{~V} / \mathrm{C}$ ratios ranging from 0.2 to 1.3 for all three strategies. Each simulation run is 30 minutes long (not including the warm-up period), and each result is averaged over 10 simulation runs, each with a different random seed.

Four performance metrics are evaluated to obtain an understanding of the solution to the mathematical program for the three strategies,

1. The furthest point of perturbation $L_{q}$.

2. Average delay for all cars $\sum_{i}\left[\left(t_{S i}-t_{O i}\right)-\frac{\overline{O S}}{v_{c}}\right] /|\{i\}|$.

3. Average delay for all buses $\sum_{j}\left[\left(t_{S j}-t_{O j}\right)-\frac{\overline{O S}}{v_{b}}\right] /|\{j\}|$.

4. The average person delay, defined in Eq.(3), assuming $X_{i}=1$, and $X_{j}=20,40,60,80$ respectively.

Based on some preliminary results, when the intersection becomes over-saturated (V/C ratio higher than 1), the perturbation quickly reaches further than $500 \mathrm{~m}$ regardless of the strategy. However, a well-functioning intersection would normally be designed to not allow large queue spillbacks. Therefore, assuming long periods of over-saturated demands is not very realistic. Rather, the intersection is designed with an average peak hour $\mathrm{V} / \mathrm{C}$ ratio of 0.8 , and larger demands are only included as statistical fluctuations for short periods (e.g. 
10 minutes). For all $\mathrm{V} / \mathrm{C}$ ratios higher than 0.8 , the simulations are only run with an over-saturated demand greater than 0.8 for the first 10 minutes, after which the base peak hour $\mathrm{V} / \mathrm{C}$ ratio of 0.8 is used for the remaining 20 minutes. Due to this, the analysis is divided into a low demand range (V/C ratio up to 0.8) and a high demand range ( $\mathrm{V} / \mathrm{C}$ ratio over 0.8$)$.

The effects of the three strategies on the three performance metrics (the furthest point of perturbation, the average car delay and the average bus delay) under different car demands are first discussed. Then, the average person delay is calculated. Influence of the number of car lanes, bus frequency, and signal timing are also analyzed.

Figure 3 summarizes the first three performance metrics described above for $\mathrm{V} / \mathrm{C}$ ratios $0.2-1.3$ assuming a bus headway of 3 minutes/bus.

[Figure 3 about here.]

\subsection{Criteria 1 - furthest distance of perturbation is less than $500 m\left(L_{q} \leq L_{q}^{\max }\right)$}

Looking at Figure $3 \mathrm{p}$, the perturbation reaches $500 \mathrm{~m}$ for the continuous bus lane strategy once the $\mathrm{V} / \mathrm{C}$ ratio exceeds 0.5 . According to criteria 1 , the continuous bus lane strategy is therefore dropped from discussion beyond this car demand. Also, the perturbation reaches $500 \mathrm{~m}$ for the pre-signal strategy once the V/C ratio exceeds 1 . Therefore, for this case, the interrupted bus lane strategy should be chosen for all over-saturated demands.

\subsection{Criteria 2 - minimum average person delay (minimize d)}

Considering car and bus delays separately, Figure $3 \mathrm{~b}$ shows that, as expected, the interrupted bus lane strategy always results in the smallest average car delay. Only minor increases in car delay are observed when a pre-signal strategy is implemented. In contrast, the continuous bus lane strategy results in much larger car delays compared to the other two strategies.

On the other hand, Figure 3c shows that the continuous bus lane strategy always results in the smallest average bus delay, with only very minor increases in bus delay observed when a pre-signal strategy is implemented (see the zoomed-in part). In contrast, the interrupted bus lane strategy results in much larger bus delays compared to the other two strategies.

Using the results presented in Figure 3 , the average person delay was also calculated. It was found that the continuous bus lane strategy results in the lowest average person delay only for very large bus occupancies or frequencies (occupancy ratio greater than 80 or frequency higher than 1 bus/minute). Such extreme conditions will only arise during peak hours. However, implementing the continuous bus lane strategy during peak hours would result in fast growing queues. Therefore, according to criteria 1 and 2 , the presignal strategy is always chosen over the continuous bus lane strategy. The continuous bus lane strategy is hence dropped from further discussion. Figure 4 summarizes the average person delay for the pre-signal strategy and the interrupted bus lane strategy for four levels of bus to car occupancy ratios.

[Figure 4 about here.]

Figure 4 shows that the interrupted bus lane strategy results in the lowest average person delay when the demand is near capacity ( $\mathrm{V} / \mathrm{C}$ ratios between 0.9 and 1.1). This results in an upper and lower bound on the $\mathrm{V} / \mathrm{C}$ ratio for the application of the interrupted bus lane strategy. It is also observed that as the occupancy ratio increases, the lower bound for the interrupted bus lane application range increases while the upper bound decreases. This implies that as the occupancy ratio increases, the pre-signal strategy is applicable for a wider range of car demands. The bounds of application based on criteria 2 are summarized in Figure 5 
[Figure 5 about here.]

When criteria 1 and 2 are both considered, it can be easily deduced that only the lower bound on $\mathrm{V} / \mathrm{C}$ for the boundary condition still remains. This is illustrated by the solid line in Figure 5 .

\subsection{Sensitivity to number of lanes ( $\lambda$ )}

Previous discussion assumes $\lambda=2$ with one car lane and one bus lane upstream of the pre-signal. Using a pre-signal on roads with more than one car lane could: i) increase the storage capacity, hence reduce the reach of the perturbation, or ii) stop cars on more lanes which could result in a larger overall person delay. The net result is unclear. Therefore, it is necessary to investigate the effects more carefully.

To analyze the impact of the number of car lanes, a bus headway of 3 minutes/bus is assumed. The patterns associated with the number of car lanes are independent of bus frequency. The cases where $\lambda=3$ or 4 are tested. Figure 6 summarizes the lower and upper bounds of application (similar to Figure 5 for the cases with one car lane, two car lanes, and three car lanes, respectively. The bounds are solely based on criteria 2 (minimizing average person delay), and when considering criteria 1 and 2 together, only the lower bounds would remain.

[Figure 6 about here.]

Results confirm our hypothesis above. When there are three car lanes, the pre-signal strategy only marginally performs better than the interrupted bus lane strategy for a small range of demand in terms of average person delay.

\subsection{Sensitivity to bus frequency $\left(f_{m}\right)$}

Previous discussion assumes a bus headway of 3 minutes/bus. Larger bus headways could: i) result in less frequent disruption to the car discharge, or ii) lead to fewer buses enjoying priority. The net result is unclear. Therefore, it is necessary to investigate the effects more carefully.

To analyze the impact of bus frequencies, one car lane is assumed because Section 5.3 shows that the presignal is most beneficial in this scenario. The interrupted bus lane and pre-signal strategies are tested for 10 different bus frequencies with headways between 0.5 minute and 15 minutes. Figure 7 summarizes the lower and upper bounds of application (similar to Figure 5 for three selected bus frequencies with headways of 1 minute, 3 minutes and 10 minutes, respectively. The bounds are solely based on criteria 2 (minimizing average person delay). Notice that as before, when considering criteria 1 and 2 together, only the lower bound would remain.

[Figure 7 about here.]

When the bus frequency is high (for example with a headway of 1 minute), the bounds are very sensitive to the occupancy ratio. In particular, for low occupancy ratios, the pre-signal strategy is almost never applicable. For high occupancy ratios, the pre-signal strategy should almost always be implemented. When the bus frequency is higher than 1 bus/minute, the pre-signal strategy is only applicable for occupancy ratios greater than 60. Therefore, for most urban intersections, the pre-signal strategy is deemed infeasible. On the other hand, when the bus frequency is low (for example with a headway of 10 minutes), the bounds become independent of the occupancy ratio. Moreover, the advantage (person delay saving) of the pre-signal also starts to diminish. For bus frequencies lower than 0.1 bus/minute, the pre-signal does not provide any meaningful savings ( 0.1 second/person) and is hence not applicable. 


\subsection{Sensitivity to signal timing ( $G$ and $\tau$ )}

Previous discussion assumes a cycle length, $\tau$, of $60 \mathrm{~s}$ and an effective green time, $G$, of $20 \mathrm{~s}$. It is unclear how different signal timings would affect the results. To analyze this, additional cycle lengths of $90 \mathrm{~s}$ and 120 s are also tested. For each cycle length, effective green ratios $(G / \tau)$ of $1 / 3,1 / 2$, and $2 / 3$ are tested. Therefore, in total nine different cases are considered, including the original signal timing. Note that for each signal timing, the $\mathrm{V} / \mathrm{C}$ ratio is calculated based on the effective discharge rate $\mu_{\text {eff }}$ of the respective setting (i.e., with different signal settings, the same $\mathrm{V} / \mathrm{C}$ ratio would correspond to different demand rates), and the location of the pre-signal $\overline{P S}$ could be different.

There are three main observations. Firstly, changing the signal timing does not seem to have an observable effect on the boundary condition based on criteria 2 (minimizing average person delay). In other words, the bounds of application in Figure 5 do not appear to be sensitive to the signal timing. Secondly, when the red time duration at the main signal is longer, the difference in average person delay between the two strategies become larger. In other words, when the demand is below the lower bound in Figure 5, the benefits provided by the pre-signal become larger with a longer red duration at the main signal. Note that the red time duration is longer when either the cycle length is longer or the effective green ratio is smaller. Therefore, out of the nine signal timings tested, it is observed that the pre-signal strategy provides the largest average person delay savings compared to the interrupted bus lane strategy when the cycle length is 120 s and the effective green time duration is 40s, and the smallest delay savings when the cycle length is $60 \mathrm{~s}$ and the effective green time duration is 40s. This is illustrated in Figure 8 for a case with $\mathrm{V} / \mathrm{C}$ ratio 0.6 and occupancy ratio 40. Lastly, for a road configuration with one car lane and one bus lane, when $G>1 / 2 \tau$, the intersection is no longer the bottleneck of the road section. This is expected since, on average, one car lane upstream of the pre-signal cannot saturate two car lanes at the main signal for more than half of the cycle length. To summarize, overall results show that when the red time duration at the main signal is long, installing a pre-signal could provide significant benefits for lower car demands.

[Figure 8 about here.]

\subsection{Summary of boundary conditions}

The analysis above illustrates the boundary conditions are of the following form: for the applicability of the pre-signal, the car demand has an upper bound, the bus to car passenger occupancy ratio has a lower bound, and the bus frequency has both a lower and an upper bound.

$$
d_{\min }=\left\{\begin{array}{l}
d(1), \text { if }\left\{\begin{array}{l}
\alpha_{m} \leq \alpha_{\max }\left(f_{m}, \beta_{m}\right) \\
f_{\min } \leq f_{m} \leq f_{\max } \\
\beta_{\min } \leq \beta_{m}
\end{array}\right. \\
d(2), \text { otherwise }
\end{array}\right.
$$

Hence, it is not optimal to operate a pre-signal continuously, as all three parameters could vary significantly throughout the day. Specifically, the results show that the bounds on car demand depend on the bus frequency and occupancy. In general, the interrupted bus lane strategy should be adopted (i.e. the presignal is continuously green) when car demand is high. The exact boundary values for a two-lane road are summarized in Table 3. For car demands exceeding the boundary values, the pre-signal should be continuously green and the interrupted bus lane strategy should be adopted. Based on these results, an adaptive control algorithm which switches between the two strategies is proposed in the next section.

[Table 3 about here.] 
Note that similar results were previously obtained analytically in (Guler and Menendez, 2014a), where a three-lane road, a fixed bus headway of 2-3 minutes/bus, a cycle length of 80 seconds and main signal red duration of 40 seconds were assumed. With the simulation approach, the boundary values could be obtained for any general scenario with any combination of bus and car demands as illustrated in Table 3 . That said, for the same test scenarios, the results from the simulation are close to those from the analytical model. For some cases, the cut-off car demand is a little lower and hence the pre-signal strategy is applicable to a smaller range of car demands than what was predicted with the analytical model. This is not surprising, however, because most factors previously excluded from the analytical model, such as bounded acceleration, lane change, reaction time, etc., worsen the pre-signal performance in reality.

\section{Second level control algorithm: combining multiple strategies}

This section first proposes an adaptive control algorithm for the pre-signal. Then, the adaptive control algorithm is tested with three case study demand profiles. The performance of this adaptive control algorithm is compared with that of a static interrupted bus lane and a continuous bus lane. Finally, the robustness of the control algorithm is examined. The goal is to determine the required accuracy level of the inputs for the proposed adaptive control algorithm to be effective.

\subsection{Guidelines for the adaptive control algorithm}

The idea of the adaptive control algorithm is to switch between different strategies at the intersection under different demands. The goal is to minimize average person delay at the intersection without causing excessive negative impacts upstream. The necessary infrastructure and relevant parameters are listed below.

Required infrastructure:

- Main signal: The main signal at the intersection operates as normal.

- Pre-signal: The pre-signal is to be built upstream of the main signal. The exact distance upstream of the main signal is given by Eq. (4). The first level control algorithm for its operation is given in Figure 2 .

- Clear markings between the main signal and the pre-signal: Irrespective of the strategy at the intersection, all lanes in this space are shared by cars and buses and should be clearly marked.

- Bus lane: The bus lane upstream of the pre-signal should be clearly marked.

Required monitoring devices:

- Traffic flow detectors: Vehicle detectors (e.g. loop detectors, cameras, radars) to measure real time traffic volume should be placed on the car lanes and the bus lane as far upstream as possible.

- Bus passenger occupancy detection: The bus passenger occupancy should be reported for all buses arriving at the intersection, either from in-bus real time occupancy detection technology, or from historical statistics. An estimate of the average car passenger occupancy should also be available, so the bus to car occupancy ratio can be calculated.

Note that if some real time monitoring devices are not available or precise, historical statistics could be used for predictions. The exact prediction method is not the focus of this research. This paper assumes perfect real time detection in the analysis and later discusses the effects of inaccurate inputs.

Pre-determined parameters: 
- Main signal capacity $\left(\mu_{e f f}\right)$ : Effective discharge capacity of the main signal.

- All necessary pre-signal operation parameters as prescribed in (Guler and Menendez (2015)). These include: the duration of the red signal during a normal cycle $\left(R_{p}\right)$, the duration of the red signal when a bus is detected $\left(R_{b}\right)$, and the red signal advance time $\left(\tau_{\Delta}\right)$.

- Table with the boundary values determined by either empirical modification of Table 3 or a similar simulation with different values for parameters in Table 2.

The length of the strategy period $\Omega$ should be carefully defined. On one hand, a short $\Omega$ is reasonable, as the free flow travel time from the detector location $O$ to the pre-signal location $P$ is short. On the other hand, the bus and car demands could fluctuate significantly between short periods, so the average result over a longer $\Omega$ gives a more stable input. For the purpose of this paper, to ensure smooth traffic operation, a larger strategy application period $\Omega$ is used (10 minutes). Also, since the actual demand is unknown at the time of decision making, a predicted demand, $\alpha_{m}^{\prime}$ for $\left[t_{m, 0}, t_{m, N}\right]$ based on previously detected demand $f\left(\alpha_{m-1}, \alpha_{m-2}, \alpha_{m-3} \ldots\right)$ is used. For simplicity, in this paper it is assumed that $\alpha_{m}^{\prime}=\alpha_{m-1}$. However, a more complex prediction method such as a linear extrapolation, a moving average or those in (Hans et al., 2015; Liu and Ma, 2009, Liu et al., 2012) could be used. The adaptive control algorithm is summarized in the flow chart in Figure 9.

[Figure 9 about here.]

\subsection{Case studies of the adaptive control algorithm}

The above online control algorithm is tested with three case study demand profiles between 06:00 and 19:30 as shown in Figure 10. This time period includes the morning peak hours (07:00-09:00), the evening peak hours (16:30-18:30), and the off-peak hours in between.

[Figure 10 about here.]

Two demand profiles represent high demand scenarios. High demand profile 1 assumes a constant high base $\mathrm{V} / \mathrm{C}$ ratio of 0.8 , whereas high demand profile 2 assumes more volatility in the demand by including statistical fluctuations for 10-minute periods during which the demand is even higher. Also, a low and under-saturated demand scenario which could arise at intersections outside the city center is tested.

For all three demand profiles, during peaks hours, the buses are assumed to arrive with a headway of 2 minutes/bus and a passenger occupancy of 60 . During off-peak hours the buses are assumed to arrive with a headway of 6 minutes/bus and a passenger occupancy of 20. Cars are assumed to have only one passenger at all times.

It is observed in the simulation that the pre-signal is continuously green (i.e. the interrupted bus lane strategy is adopted) between 7-9 and 16-19. This is expected from the adaptive control algorithm because during the peak hours the bus headway is 2 minutes/bus, with an occupancy of 60 people/bus, so the V/C ratio boundary value of 0.6 (see Table 3 ) is exceeded.

According to the adaptive control algorithm, the pre-signal at the intersection is always on if the low demand profile is used. This is a general characteristic when a pre-signal is installed at an intersection with a low demand. The advantage of implementing an adaptive control algorithm for the pre-signals at these intersections (compared to leaving the pre-signal continuously on) is the ability to respond to occasional high demands (for example due to re-routing or special events). 


\subsection{Comparison with the continuous bus lane strategy and the interrupted bus lane strategy}

The purpose of this section is to compare the effects of the other two static strategies to that of a pre-signal with the proposed adaptive control algorithm (second level control). The road configurations of these three strategies are as illustrated in Figure 1 .

Two traffic performance metrics are examined. First, to reflect the level of traffic congestion, the average car occupancy of a loop detector placed $1 \mathrm{~km}$ upstream of the pre-signal location is used. Second, the average person delay is calculated. These two traffic performance metrics are compared across the three operating strategies for each of the tested demand profiles in Figure 10. The averages for the performance metrics are calculated and compared for the peak and off-peak hours respectively. The results are summarized in Table 4.

[Table 4 about here.]

Implementing the continuous bus lane strategy is detrimental to the rest of the network, as it causes serious traffic congestion. This is illustrated in Tables $4 \mathrm{a}$ and Table $4 \mathrm{~b}$ with the high detector occupancy, especially during peak hours. In addition, the continuous bus lane strategy results in the highest average person delay, especially in the peak hours, despite the priority the buses receive. In contrast, the pre-signal with the proposed adaptive control algorithm does not cause extra stress to the rest of the network as the average detector occupancy is only a little higher than when the interrupted bus lane strategy is implemented. Moreover, installing the pre-signal with the proposed adaptive control algorithm at an intersection results in the least average person delay under all scenarios. These results demonstrate that implementing a pre-signal with the proposed adaptive control algorithm improves the operation of the intersection. Public transport priority is provided while traffic control goals are better achieved.

\subsection{Robustness testing}

In this section the authors study the robustness of the proposed adaptive control algorithm, i.e. its sensitivity to the data quality of the traffic inputs. This is relevant because in practice, real time data could be inaccurate or incomplete, and average historical data does not reflect daily fluctuations. Therefore, the input values for the adaptive control algorithm could be an under/over-estimation. Nevertheless, the adaptive control algorithm should have a relatively stable performance despite these uncertainties.

The proposed adaptive control algorithm requires three real time inputs: the traffic demand prediction $\left(\alpha_{m}^{\prime}\right)$, the bus frequency $\left(f_{m}\right)$ and the bus to car occupancy ratio $\left(\beta_{m}\right)$. Among these three, the bus frequency is relatively accurate because it only requires detection of buses on the bus lane, for example by a loop detector, and is usually based on a schedule. The traffic demand, however, could be inaccurate because it is a prediction and is assumed to be constant over the strategy period $\Omega$. The bus occupancy is usually difficult to detect in real time and most likely the algorithm could only use historical statistics. The performance of the pre-signal could be affected because the control algorithm would not switch the pre-signal operation on and off at the correct time.

Therefore, the robustness of the proposed adaptive control algorithm is important. It is tested against the uncertainties in traffic volume and bus occupancy. The idea is to examine if implementing a pre-signal with the proposed adaptive control algorithm can still reduce average travel time, when the input values to the algorithm are different from the actual traffic situation.

The adaptive control algorithm uses the same input values as in the high demand profile 1 in Figure 10. However, the actual traffic (i.e. the demand and the bus occupancy) is simulated slightly differently. Four cases are studied for each of the two parameters: 
1. The simulated value fluctuates around the true value with a normally distributed random error. This represents a random error.

2. The simulated value is constantly higher than the true value. This represents a systematic overestimation.

3. The simulated value is constantly lower than the true value. This represents a systematic underestimation.

4. The simulated value lags behind the true value. This represents a systematic lag of input values.

The lower and higher values simulated for the traffic volume to capacity ratio are 0.1 under or over the input $\mathrm{V} / \mathrm{C}$ ratios, representing a discrepancy of $12-33 \%$. The random error simulated in the fluctuating profile has a standard deviation of 0.05 . The lower and higher values simulated for the bus occupancy are 10 people under or over the input bus occupancies, representing a discrepancy of $17-50 \%$. The random error simulated in the fluctuating profile has a standard deviation of 5. For both the traffic volume and the bus occupancy, the lag profile is 10 minutes behind the actual value. This implies that the changes to the demand can only be detected after multiple cycles. A relatively large lag is used in this case because the demand profile is relatively smooth, so a smaller lag would not have much effect on the results. In reality, the demand is likely less smooth than that assumed here, but the lag in detecting the changes in demand should be much smaller too. Figure 11 summarizes the simulated traffic demand and occupancy profiles.

[Figure 11 about here.]

Each case is applied independently (i.e. everything else is assumed to remain unchanged). Therefore, in total 8 types of discrepancies are tested. Simulation results show that, a pre-signal with the adaptive control algorithm still provides person delay savings compared to the interrupted bus lane strategy, as summarized in Figure 12 .

[Figure 12 about here.]

It can be seen from Figure 12 that although an inaccurate input generally reduces the benefits of implementing an adaptive pre-signal, the reduction is at most $20 \%$ for the studied cases. The results are most sensitive to inaccurate demand inputs because the pre-signal will be operating with less than optimal timings. However, detecting demand is relatively easy with modern technologies, for example with loop detectors, cameras, or radars. Admittedly, there could be a lag of the detected demand from the arriving demand, because detected values from the previous cycle are used to calculate pre-signal timing for the next cycle. However, results show that this lag would not affect the results too much. Bus occupancy, on the other hand, is usually difficult to detect in real time, but it is shown here that all four error types in bus occupancy value have little effect on the average person delay savings. Therefore, it could suffice to use historical occupancy statistics as algorithm inputs. Overall, although this is not a systematic sensitivity analysis, the case studies show clearly that for a reasonable range of inaccuracies, the savings in average person delays are not significantly affected. Therefore, the proposed adaptive control algorithm is robust and does not rely on very exact inputs.

\section{Conclusions}

This paper developed an adaptive control algorithm for implementing a pre-signal at an isolated urban intersection. The goal of the adaptive control algorithm is to minimize average person delay at the intersection without causing excessive negative impacts upstream. The adaptive control algorithm needs only three inputs, namely the traffic volume, the bus frequency, and the bus occupancy. These could be obtained as a combination of real time detection and historical statistics. The necessary infrastructure and relevant 
parameters were listed, and guidelines for implementation were given. Compared to using a static continuous bus lane strategy at an intersection, implementing a pre-signal has much less negative impact upstream because of a higher discharging capacity. Compared to using a static interrupted bus lane strategy at an intersection, implementing a pre-signal encourages public transport by providing priority to buses. It was demonstrated that, under all scenarios, implementing a pre-signal with the proposed adaptive control algorithm (with the second level control) will result in the least average person delay at the intersection compared to using a static interrupted bus lane or a static continuous bus lane.

The adaptive control algorithm switches between the pre-signal strategy and the interrupted bus lane strategy (note that the continuous bus lane strategy was found never to be beneficial in terms of average person delay savings). It was illustrated that such switches are easy to implement. The switches only require to change the red and green durations of the pre-signal. These are either determined with the first level control algorithm (for the pre-signal strategy) or the pre-signal remains always green (for the interrupted bus lane strategy). Switching between these two operations would lead to minimum (if any) driver confusion since the configuration of the roadway would not change. Compared to implementing a static pre-signal strategy, the adaptive control algorithm can tailor operations to varying traffic demands throughout the day. The algorithm is expected to function well with a wide range of car demands, bus frequencies, and bus occupancies. Moreover, the proposed algorithm is robust and does not rely on very exact inputs. Hence, when real time inputs are not available, historical data could be used.

The intersection model in the paper is relatively simple as all cars and buses are assumed to drive through the intersection. Nevertheless, having a mixture of vehicle movements should not significantly change the main conclusions. For example, left/right-turning buses would be able to complete their movements in a similar manner to through moving buses, except for the additional delay which they would encounter even if they had a dedicated lane. Moreover, the existence of a pre-signal would facilitate the bus changing lanes from the right-most to the left-most hence would provide further delay savings over the interrupted bus lane strategy. On the other hand, right-turning cars could queue differently from through moving vehicles. In this case, the pre-signal can facilitate the bus to change lanes in order to skip the queue of right-turning vehicles which may remain even after the queue of through moving vehicles at the main signal clears. Again, this could provide bus delay savings over the interrupted bus lane strategy. Left-turning cars could affect the discharge rate at the main signal if there were no separate left-turning phase, as two different queues might form at the main signal depending on the mix of the car movements. If a large percentage of cars desire to turn left, the red pre-signal should be even shorter, so that there are enough through moving cars queued at the main signal to saturate it. If a separate left-turning phase exists, this phase could precede or follow the through movement phase. When the left-turning phase is before the main phase, left-turning cars need to be discharged earlier to queue on the left lane in front of the main signal. Therefore, vehicles need to be separated by movement before the pre-signal, and at least two car lanes at the pre-signal would be desirable. The left-turning phase could also be designed to be after the main phase. In this case, the left-turning vehicles will be queuing on the left lane while the main signal is discharging with one fewer lane. In this case, the pre-signal red duration could likely be extended, because the effective discharge rate of the main signal is lower. In any case, installing a pre-signal could still provide priority to buses without causing excessive delay to cars.

Methodologically, studying a pre-signal with a simulation model added to analytical models because previous analytical formulations could not account for factors such as bounded acceleration, reaction time, and lane changes, whereas the stochastic variation of many factors are included in the simulation. Various performance metrics of the pre-signal operation could be examined and compared to other strategies using a realistic demand. Hence, the boundary conditions could be established for any general scenario.

Lastly, the micro-simulation model built the foundation to study pre-signal operations on the arterial and network level in future research, which are more difficult to examine analytically. The ultimate goal is to identify the optimal operating strategy for pre-signals at the network level so that a good balance between providing bus priority and sustaining car throughput in a city could be achieved. As many cities in the world 
aim to both encourage public transport and reduce congestion, implementing pre-signals could be a viable solution.

\section{Acknowledgement}

This research has been partially funded by the Swiss National Science Foundation (SNSF) under the project name SignBus, contract 205121_150180.

\section{References}

\section{References}

Department for Transport (U.K.), 2003. Traffic Control System Design for All Purpose Roads (Compendium of Examples), 1st Edition. Highways agency.

Forschungsgesellschaft für Straßen-und Verkehrswesen, 1991. Merkblatt für Maßnahmen zur Beschleunigung des ÖPNV. FGSV, Köln, Germany.

Guler, S. I., Gayah, V. V., Menendez, M., 2015. Providing bus priority at signalized intersections with single-lane approaches. Transportation Research Procedia 9, 225-245.

Guler, S. I., Gayah, V. V., Menendez, M., 2016. Bus priority at signalized intersections with single-lane approaches: A novel pre-signal strategy. Transportation Research Part C: Emerging Technologies.

Guler, S. I., Menendez, M., 2013. Empirical evaluation of bus and car delays at pre-signals. In: Swiss Transport Research Conference (STRC), Ascona, Switzerland.

Guler, S. I., Menendez, M., 2014a. Analytical formulation and empirical evaluation of pre-signals for bus priority. Transportation Research Part B: Methodological 64, 41-53.

Guler, S. I., Menendez, M., 2014b. Evaluation of presignals at oversaturated signalized intersections. Transportation Research Record: Journal of the Transportation Research Board (2418), 11-19.

Guler, S. I., Menendez, M., 2015. Pre-signals for bus priority: basic guidelines for implementation. Public Transport, 1-16.

Hans, E., Chiabaut, N., Leclercq, L., 2015. Applying variational theory to travel time estimation on urban arterials. Transportation Research Part B: Methodological 78, 169-181.

He, H., Guler, I., Menendez, M., 2014. The effects of pre-signals at an isolated intersection: simulation results.

Liu, H. X., Ma, W., 2009. A virtual vehicle probe model for time-dependent travel time estimation on signalized arterials. Transportation Research Part C: Emerging Technologies 17 (1), 11-26.

Liu, H. X., Ma, W., Wu, X., Hu, H., 2012. Real-time estimation of arterial travel time under congested conditions. Transportmetrica 8 (2), 87-104.

Transport for London, 2009. Bus pre-signal assessment and design guide. Retrieved: January 2009.

URL www.tfl.gov.uk/assets/downloads/businessandpartners/Bus_Pre-Signals_Aug05.pdf

Von Stein, W., 1961. Traffic flow with pre-signals and the signal funnel. Theory of Traffic Flow, 28-56. 
Wu, J., Hounsell, N., 1998. Bus priority using pre-signals. Transportation Research Part A: Policy and Practice $32(8), 563-583$.

Xuan, Y., Daganzo, C. F., Cassidy, M. J., 2011. Increasing the capacity of signalized intersections with separate left turn phases. Transportation research part B: methodological 45 (5), 769-781. 


\section{List of Figures}

$1 \quad$ Layout of the three strategies upstream of the intersection (not drawn to scale). . . . . . . . . 21

$2 \quad$ Flow chart for the operation of the pre-signal (first level control). . . . . . . . . . . . . . . . . 22

3 Performance metrics vs. V/C ratios. (a) furthest perturbation point; (b) average car delay; (c) average bus delay $\left(f_{m}=3 \mathrm{~min} / \mathrm{bus}, \lambda=2\right)$. . . . . . . . . . . . . . . . . . . . . 23

4 Comparison of average person delay for the pre-signal strategy and the interrupted bus lane strategy with different bus to car occupancy ratios $\left(f_{m}=3 \mathrm{~min} / \mathrm{bus}, \lambda=2\right)$. $\ldots . . . . . . .24$

$5 \quad$ Bounds of application (based only on criteria 2): the pre-signal strategy vs. the interrupted bus lane strategy $\left(f_{m}=3 \mathrm{~min} /\right.$ bus,$\left.\lambda=2\right) . \quad \ldots \ldots \ldots \ldots \ldots \ldots \ldots$

$6 \quad$ Sensitivity of bounds (based only on criteria 2) to number of lanes $(\lambda)$ : the pre-signal strategy vs. the interrupted bus lane strategy $\left(f_{m}=3 \mathrm{~min} /\right.$ bus $) . \ldots . . . . . . . . . .26$

$7 \quad$ Sensitivity of bounds (based only on criteria 2) to bus frequency $\left(f_{m}\right)$ : the pre-signal strategy vs. the interrupted bus lane strategy $(\lambda=2) . \ldots \ldots \ldots \ldots \ldots$. . . . . . . . . . . . 27

8 Pre-signal delay savings per person (seconds) compared to an interrupted bus lane option, for different signal timings $\left(f_{m}=3 \mathrm{~min} /\right.$ bus, $\lambda=2, \mathrm{~V} / \mathrm{C}=0.6$, occupancy ratio=40 $)$.

$9 \quad$ Flow chart for control algorithm to switch between strategies (second level control). . . . . . 29

$10 \quad$ The three simulated demand profiles. . . . . . . . . . . . . . . . . . . . . . . . . . . 30

11 Simulated traffic profiles for the robustness test. . . . . . . . . . . . . . . . . . . . . . . . . 31

12 Actual pre-signal person delay savings with inaccurate inputs as a percentage of expected savings with perfect information. . . . . . . . . . . . . . . . . . . . 32 

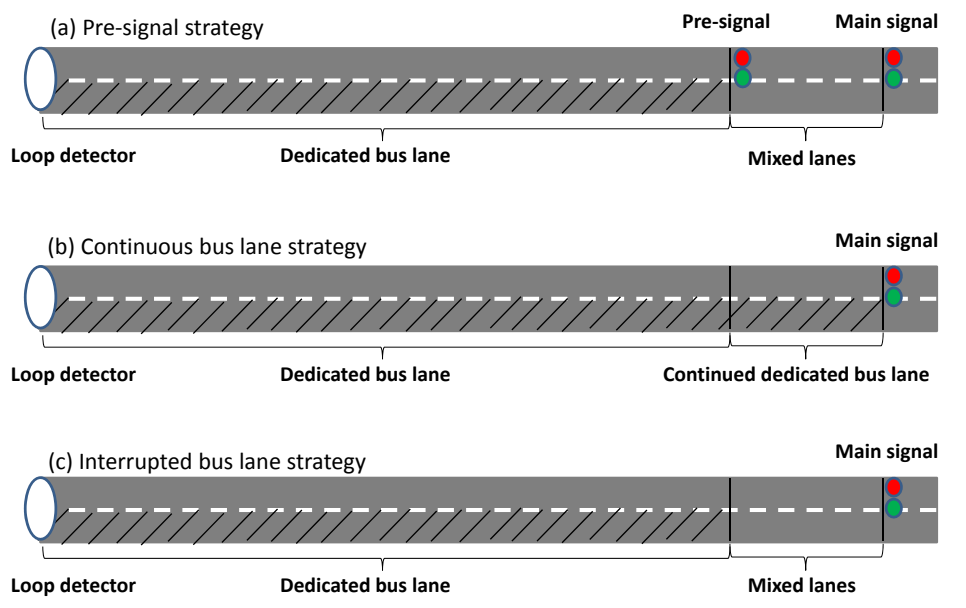

Figure 1: Layout of the three strategies upstream of the intersection (not drawn to scale). 


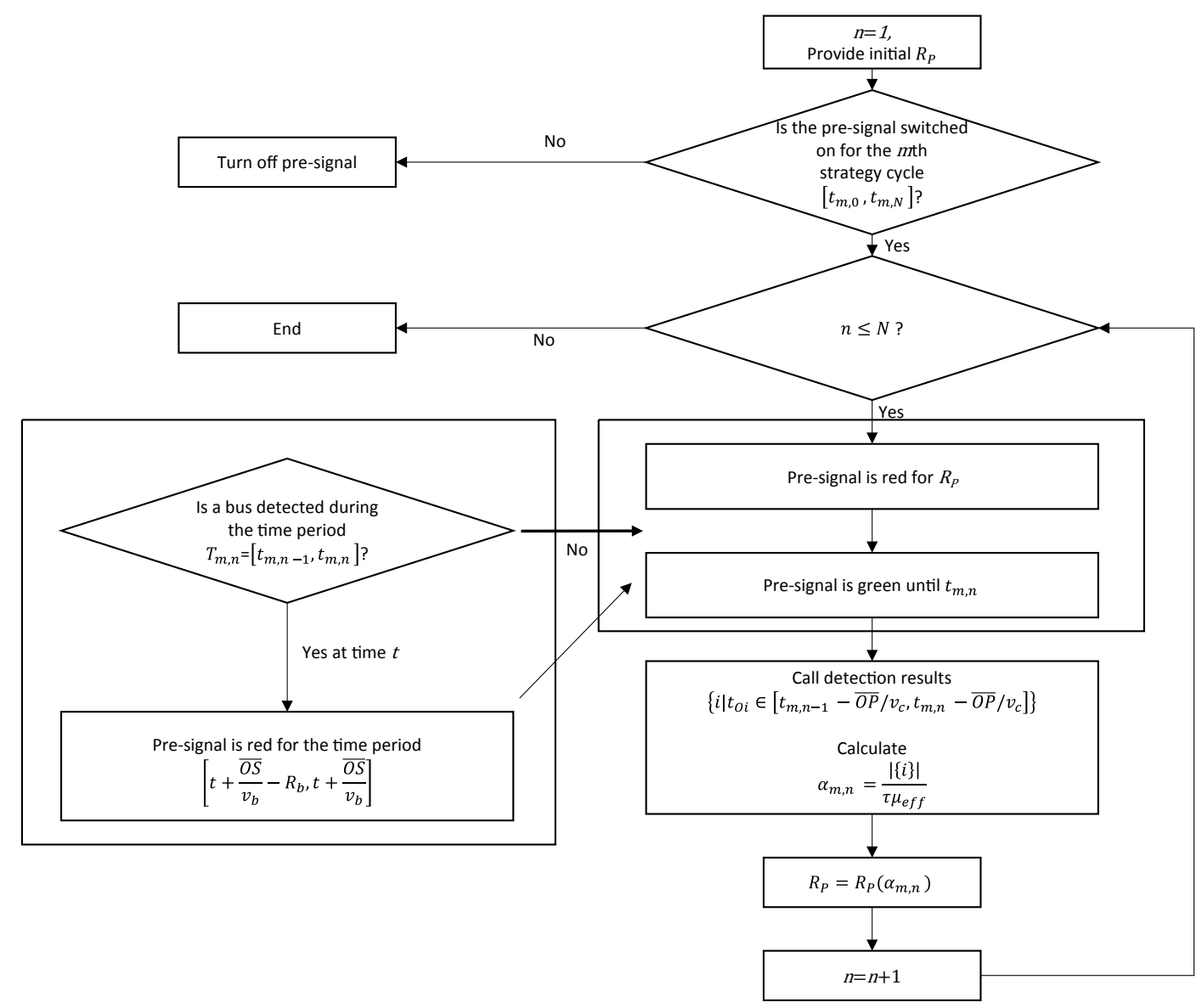

Figure 2: Flow chart for the operation of the pre-signal (first level control). 
$\rightarrow$ pre-signal $\rightarrow$-continuous bus lane $\rightarrow$-interrupted bus lane

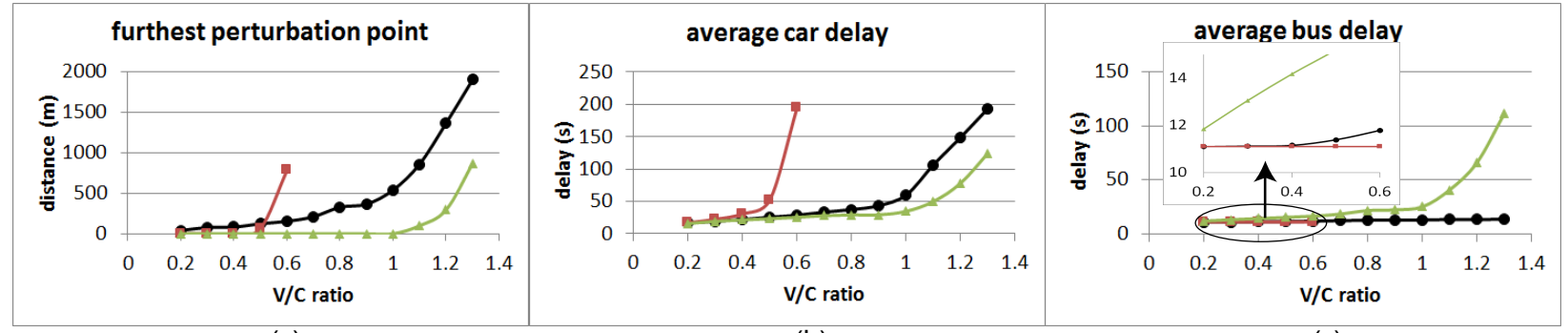

(a)

(b)

(c)

Figure 3: Performance metrics vs. V/C ratios. (a) furthest perturbation point; (b) average car delay; (c) average bus delay $\left(f_{m}=3 \mathrm{~min} /\right.$ bus, $\left.\lambda=2\right)$. 


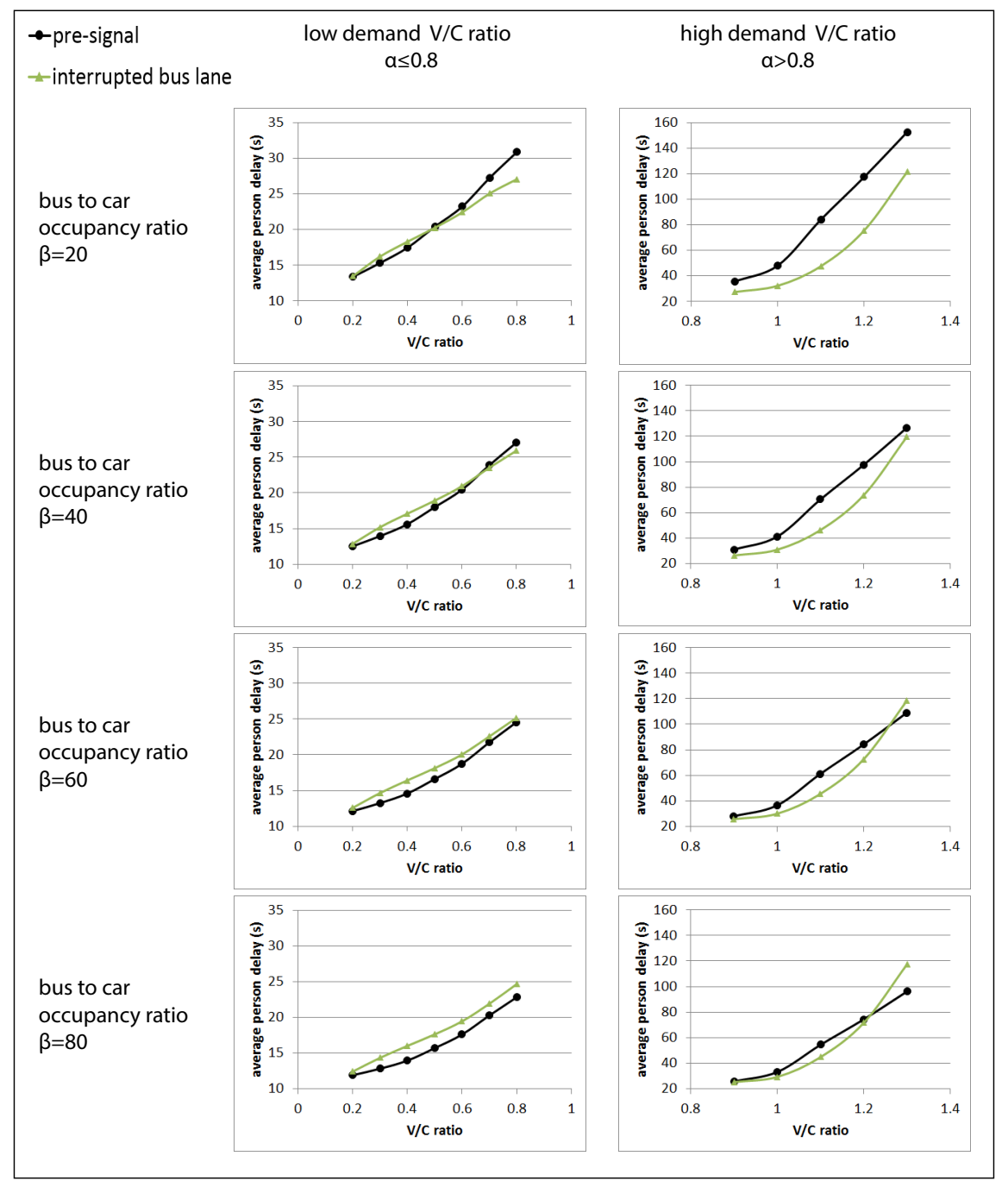

Figure 4: Comparison of average person delay for the pre-signal strategy and the interrupted bus lane strategy with different bus to car occupancy ratios $\left(f_{m}=3 \mathrm{~min} / \mathrm{bus}, \lambda=2\right)$. 


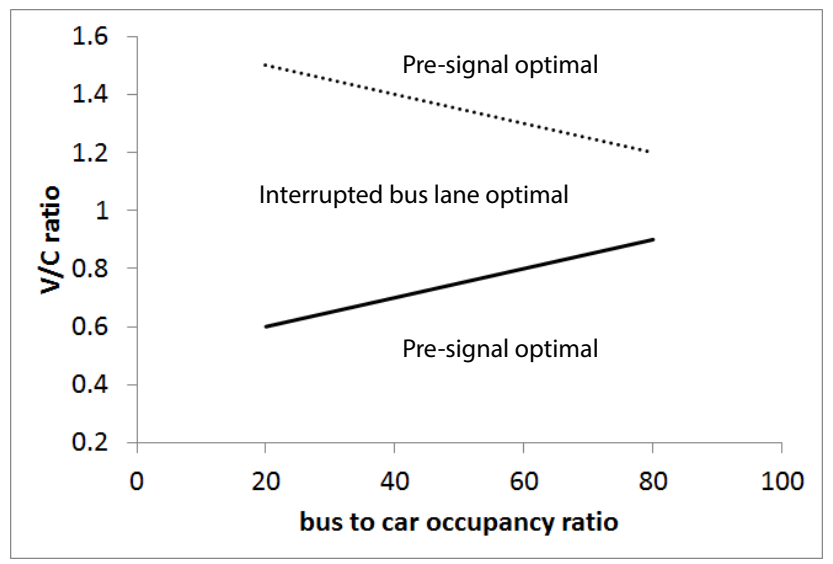

Figure 5: Bounds of application (based only on criteria 2): the pre-signal strategy vs. the interrupted bus lane strategy $\left(f_{m}\right.$ $=3 \mathrm{~min} /$ bus, $\lambda=2$ ). 


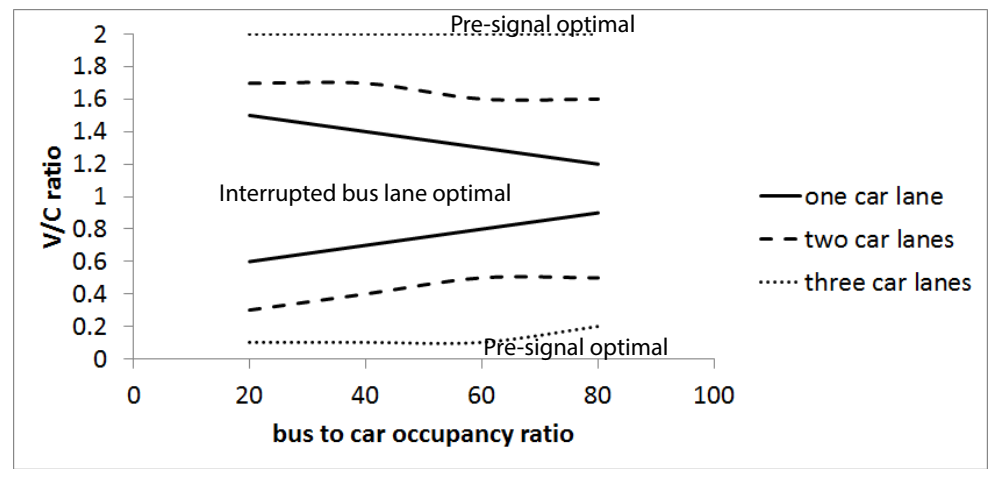

Figure 6: Sensitivity of bounds (based only on criteria 2) to number of lanes $(\lambda)$ : the pre-signal strategy vs. the interrupted bus lane strategy $\left(f_{m}=3 \mathrm{~min} /\right.$ bus $)$. 


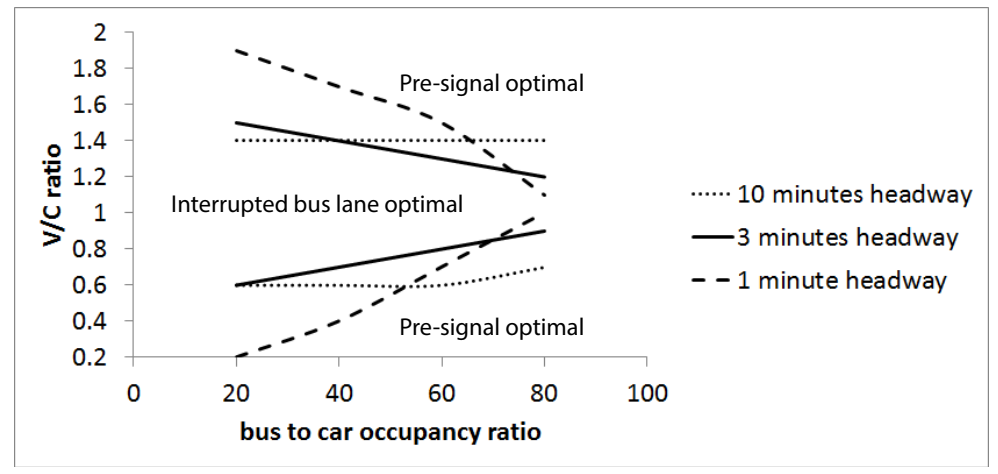

Figure 7: Sensitivity of bounds (based only on criteria 2) to bus frequency $\left(f_{m}\right)$ : the pre-signal strategy vs. the interrupted bus lane strategy $(\lambda=2)$. 


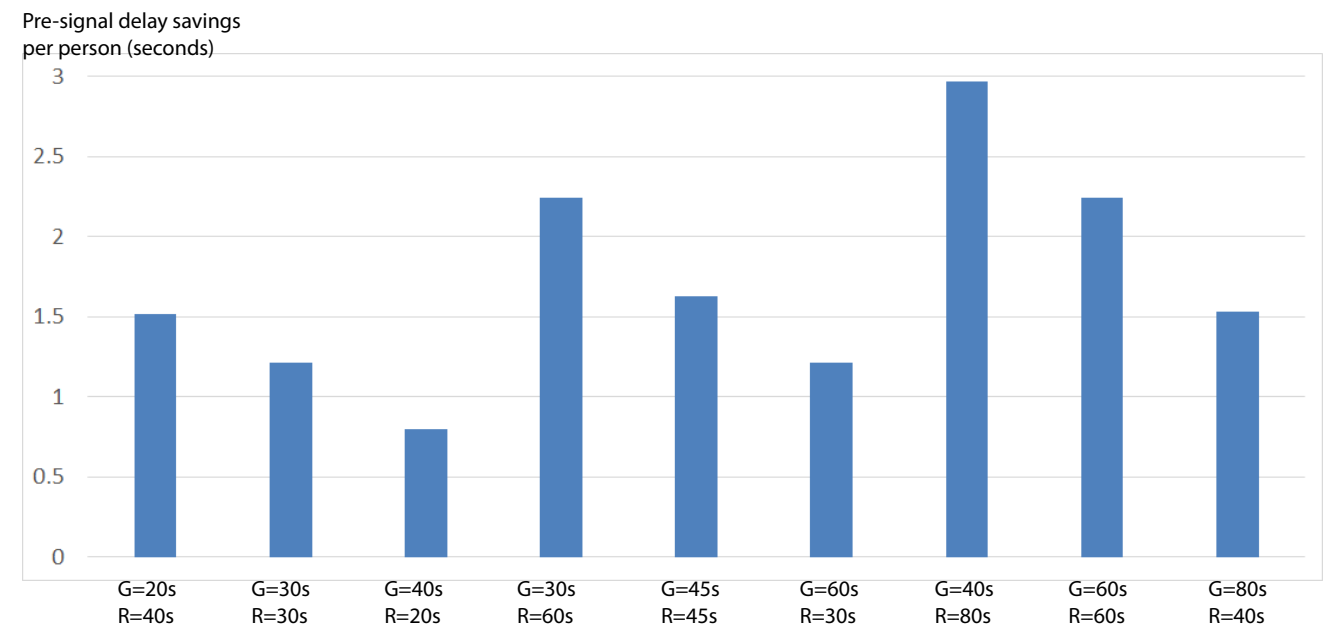

Figure 8: Pre-signal delay savings per person (seconds) compared to an interrupted bus lane option, for different signal timings $\left(f_{m}=3 \mathrm{~min} /\right.$ bus, $\lambda=2, \mathrm{~V} / \mathrm{C}=0.6$, occupancy ratio=40 $)$. 


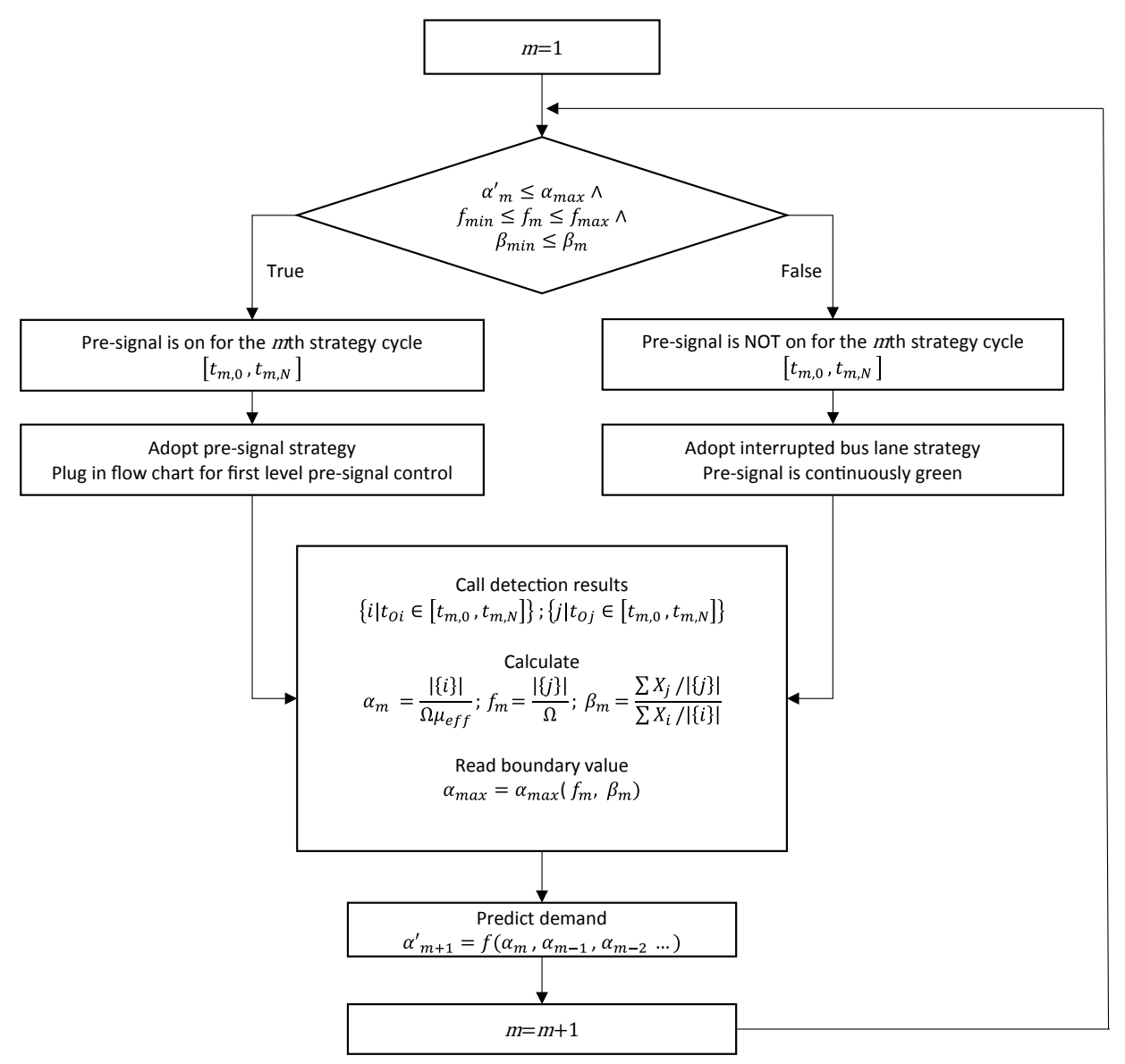

Figure 9: Flow chart for control algorithm to switch between strategies (second level control). 


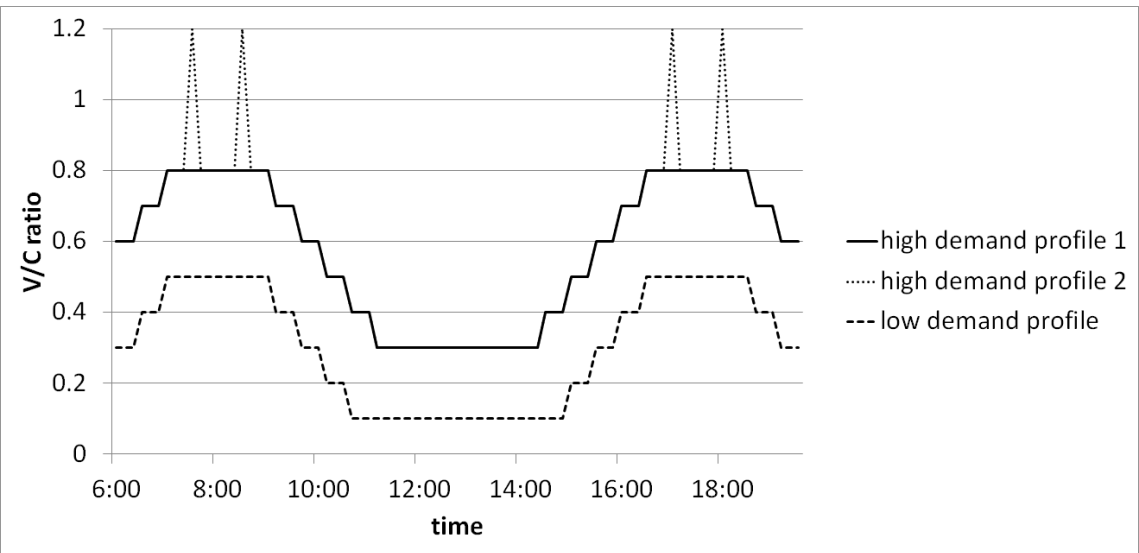

Figure 10: The three simulated demand profiles. 

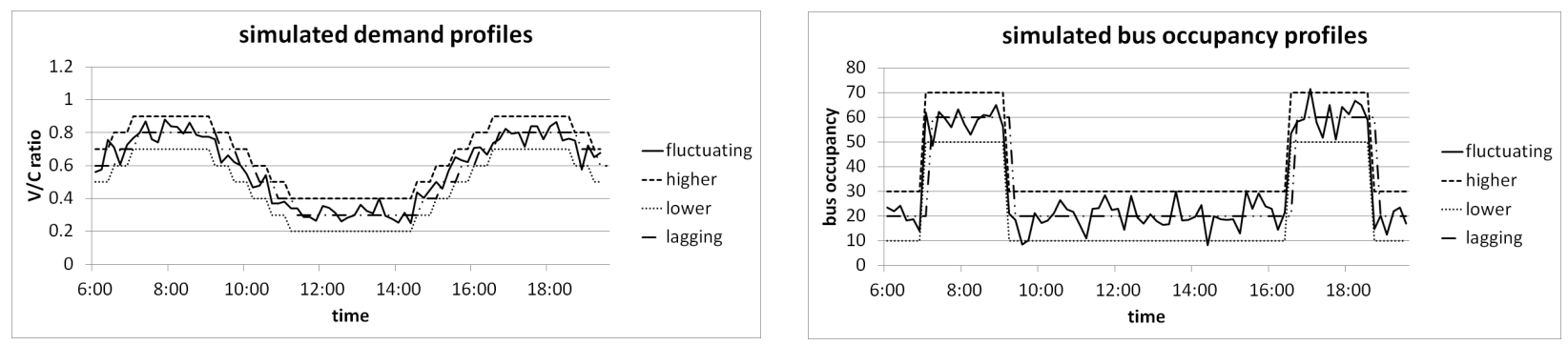

Figure 11: Simulated traffic profiles for the robustness test. 


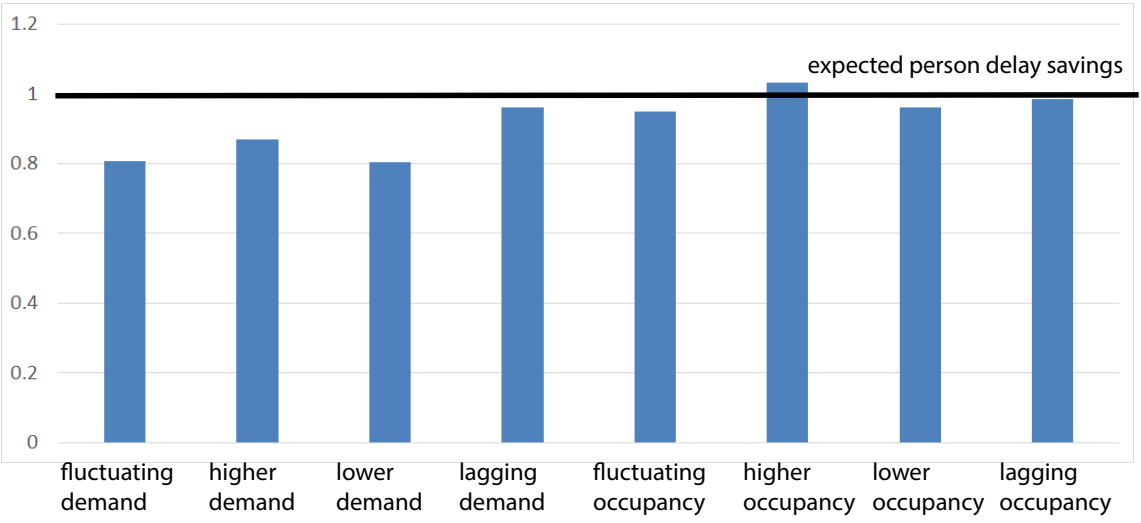

Figure 12: Actual pre-signal person delay savings with inaccurate inputs as a percentage of expected savings with perfect information. 


\section{List of Tables}

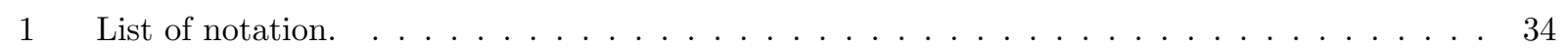

$2 \quad$ Variables used in the simulation model. $\ldots \ldots \ldots \ldots \ldots \ldots \ldots$

$3 \quad$ Boundary values $\left(\alpha_{\max }\right)$ for a two-lane road. $\ldots \ldots \ldots \ldots \ldots$

$4 \quad$ Case study results from three demand profiles. $\ldots \ldots \ldots \ldots \ldots \ldots$ 
Table 1: List of notation.

\begin{tabular}{|c|c|}
\hline \multicolumn{2}{|l|}{ Locations } \\
\hline$S$ & Main signal \\
\hline$P$ & Pre-signal \\
\hline$O$ & Loop detector \\
\hline \multicolumn{2}{|l|}{ Signal timings } \\
\hline$G$ & Effective green time duration of the main signal $[\mathrm{s}]$ \\
\hline$\tau$ & Cycle length of both signals $[\mathrm{s}]$ \\
\hline$\tau_{\Delta}$ & Advance time of pre-signal red $[\mathrm{s}]$ \\
\hline$R_{p}$ & Red time duration of the pre-signal $[\mathrm{s}]$ \\
\hline$R_{b}$ & Red time duration of the pre-signal in presence of a bus $[\mathrm{s}]$ \\
\hline \multicolumn{2}{|l|}{ Road and traffic properties } \\
\hline$\lambda$ & Total number of lanes \\
\hline$\mu$ & Capacity of a single lane $[\mathrm{veh} / \mathrm{s}]$ \\
\hline$\mu_{e f f}$ & Effective discharge capacity of the intersection [veh/s] \\
\hline$k_{j a m}$ & Jam density of a single lane $[\mathrm{veh} / \mathrm{m}]$ \\
\hline$v_{c}$ & Free-flow speed of cars $[\mathrm{m} / \mathrm{s}]$ \\
\hline$v_{b}$ & Free-flow speed of buses $[\mathrm{m} / \mathrm{s}]$ \\
\hline$L_{q}$ & Furthest distance of traffic perturbation $[\mathrm{m}]$ \\
\hline$L_{q}^{\max }$ & Maximum acceptable distance of traffic perturbation $[\mathrm{m}]$ \\
\hline$d^{4}$ & Average person delay $[\mathrm{s}]$ \\
\hline \multicolumn{2}{|l|}{ Individual vehicle properties } \\
\hline$k \in K$ & Vehicle index and the set of all vehicle indices \\
\hline$i \in I, I \subseteq K$ & Car index and the set of all car indices \\
\hline$j \in J, J \subseteq K$ & Bus index and the set of all bus indices \\
\hline$X_{k}$ & Number of people on vehicle $k$ \\
\hline$t_{O k}$ & Time at which vehicle $k$ passes loop detector \\
\hline$t_{S k}$ & Time at which vehicle $k$ passes main signal \\
\hline \multicolumn{2}{|l|}{ Algorithm inputs } \\
\hline$\Omega$ & Period length of strategy application $[\mathrm{s}]$ \\
\hline$N=\Omega / \tau$ & Number of signal cycles in each strategy application period \\
\hline$T_{m, n}=\left[t_{m, n-1}, t_{m, n}\right], 1 \leq n \leq N$ & $\begin{array}{l}\text { The } n \text {th signal cycle within the } m \text { th strategy period, } t_{m, n}-t_{m, n-1}= \\
\tau, t_{m, N}-t_{m, 0}=\Omega\end{array}$ \\
\hline$I_{m}$ & Number of cars detected in the $m$ th strategy period \\
\hline$J_{m}$ & Number of buses detected in the $m$ th strategy period \\
\hline$\alpha_{m, n}, \alpha_{m}$ & $\begin{array}{l}\text { Car volume to capacity ratio in the signal cycle }\left[t_{m, n-1}, t_{m, n}\right] \text { or the } \\
m \text { th strategy period }\left[t_{m, 0}, t_{m, N}\right] \text {, respectively }\end{array}$ \\
\hline$\alpha_{m}^{\prime}$ & Car volume to capacity ratio prediction for the $m$ th strategy period \\
\hline$f_{m}$ & Bus frequency in the $m$ th strategy period \\
\hline$\beta_{m}$ & Bus to car passenger occupancy ratio in the $m$ th strategy period \\
\hline
\end{tabular}


Table 2: Variables used in the simulation model.

\begin{tabular}{|l|l|}
\hline$\lambda$ & 2 \\
$\mu$ & $2100 \mathrm{veh} / \mathrm{h}$ \\
$\mu_{\text {eff }}$ & $1400 \mathrm{veh} / \mathrm{h}$ \\
$\tau$ & $60 \mathrm{~s}$ \\
$G$ & $20 \mathrm{~s}$ \\
$v_{c}$ & $50 \mathrm{~km} / \mathrm{h}$ \\
$v_{b}$ & $30 \mathrm{~km} / \mathrm{h}$ \\
$L_{q}^{\max }$ & $500 \mathrm{~m}$ \\
$\frac{P S}{P S}$ & $83 \mathrm{~m}$ \\
$\overline{O P}$ & $2000 \mathrm{~m}$ \\
\hline
\end{tabular}


Table 3: Boundary values $\left(\alpha_{\max }\right)$ for a two-lane road.

\begin{tabular}{|c|c|c|c|c|c|c|c|c|c|c|}
\hline$\beta_{\beta_{m}} 1 / f_{m}$ & $1 \mathrm{~min}$ & $2 \mathrm{~min}$ & $3 \min$ & $4 \mathrm{~min}$ & $5 \mathrm{~min}$ & $6 \mathrm{~min}$ & $7 \mathrm{~min}$ & $8 \min$ & $9 \min$ & $10 \mathrm{~min}$ \\
\hline 10 & 0.1 & 0.2 & 0.3 & 0.4 & 0.5 & 0.5 & 0.5 & 0.5 & 0.5 & 0.5 \\
\hline 20 & 0.2 & 0.4 & 0.6 & 0.6 & 0.6 & 0.6 & 0.6 & 0.6 & 0.6 & 0.6 \\
\hline 30 & 0.3 & 0.4 & 0.6 & 0.6 & 0.6 & 0.6 & 0.6 & 0.6 & 0.6 & 0.6 \\
\hline 40 & 0.4 & 0.5 & 0.7 & 0.7 & 0.7 & 0.6 & 0.6 & 0.6 & 0.6 & 0.6 \\
\hline 50 & 0.5 & 0.6 & 0.7 & 0.7 & 0.7 & 0.7 & 0.6 & 0.6 & 0.6 & 0.6 \\
\hline 60 & 0.7 & 0.7 & 0.8 & 0.8 & 0.7 & 0.7 & 0.7 & 0.6 & 0.6 & 0.6 \\
\hline 70 & 0.9 & 0.9 & 0.8 & 0.8 & 0.7 & 0.7 & 0.7 & 0.7 & 0.6 & 0.6 \\
\hline 80 & 1 & 0.9 & 0.9 & 0.9 & 0.8 & 0.8 & 0.8 & 0.8 & 0.7 & 0.7 \\
\hline
\end{tabular}


Table 4: Case study results from three demand profiles.

(a) High demand profile 1.

\begin{tabular}{|c|c|l|l|l|}
\hline \multicolumn{2}{|c|}{ Strategies } & $\begin{array}{l}\text { interrupted } \\
\text { bus lane }\end{array}$ & $\begin{array}{l}\text { continuous } \\
\text { bus lane }\end{array}$ & $\begin{array}{l}\text { pre-signal with second } \\
\text { level control }\end{array}$ \\
\hline \multirow{2}{*}{ Average detector occupancy $(\%)$} & Peak & 0.42 & 0.94 & 0.42 \\
\cline { 2 - 5 } & Off-peak & 0.27 & 0.64 & 0.30 \\
\hline \multirow{2}{*}{ Average person delay(s) } & Peak & 25.52 & 129.79 & 25.52 \\
\cline { 2 - 5 } & Off-peak & 18.84 & 54.6 & 17.32 \\
\hline
\end{tabular}

(b) High demand profile 2.

\begin{tabular}{|c|c|l|l|l|}
\hline \multicolumn{2}{|c|}{ Strategies } & $\begin{array}{l}\text { interrupted } \\
\text { Pus lane }\end{array}$ & $\begin{array}{l}\text { continuous } \\
\text { bus lane }\end{array}$ & $\begin{array}{l}\text { pre-signal with second } \\
\text { level control }\end{array}$ \\
\hline \multirow{2}{*}{ Average detector occupancy (\%) } & Peak & 0.61 & 0.97 & 0.61 \\
\cline { 2 - 5 } & Off-peak & 0.27 & 0.66 & 0.30 \\
\hline \multirow{2}{*}{ Average person delay(s) } & Peak & 36.91 & 138.28 & 36.91 \\
\cline { 2 - 5 } & Off-peak & 18.84 & 58.15 & 17.32 \\
\hline
\end{tabular}

(c) Low demand profile.

\begin{tabular}{|c|c|l|l|l|}
\hline \multicolumn{2}{|c|}{ Strategies } & $\begin{array}{l}\text { interrupted } \\
\text { bus lane }\end{array}$ & $\begin{array}{l}\text { continuous } \\
\text { bus lane }\end{array}$ & $\begin{array}{l}\text { pre-signal with second } \\
\text { level control }\end{array}$ \\
\hline \multirow{2}{*}{ Average detector occupancy $(\%)$} & Peak & 0.25 & 0.25 & 0.25 \\
\cline { 2 - 5 } & Off-peak & 0.11 & 0.11 & 0.11 \\
\hline \multirow{2}{*}{ Average person delay(s) } & Peak & 19.13 & 50.52 & 18.16 \\
\cline { 2 - 5 } & Off-peak & 17.45 & 17.85 & 16.84 \\
\hline
\end{tabular}

